\title{
Electromagnetic Compatibility Issues during Faults in Electric Power Systems
}

\author{
M. Buzdugan ${ }^{1}$, H. Bălan ${ }^{1}$ and H. Laslo ${ }^{2}$ \\ ${ }^{1}$ Technical University of Cluj-Napoca, \\ 28, Memorandumului str. - Cluj-Napoca, 400114 (Romania) \\ Phone/Fax number: +40 744 560833, e-mail: mircea.buzdugan@insta.utcluj.ro, horia.balan@eps.utcluj.ro \\ 2 Electroplus Ltd. \\ 152, Traian Vuia str. - Cluj-Napoca, 400397 (Romania) \\ Phone/Fax number+40 264 416716, e-mail: office@electroplus-cluj.ro
}

\begin{abstract}
The main objective of the paper is to assess the influence of power systems faults, namely short circuits, on electromagnetic compatibility compliance.

In the introductory section, the main concepts of electromagnetic compatibility are addressed, with a special stress on electromagnetic conducted emissions and interference.

The second section, devoted to experimental results, presents the measurements carried out on a $110 / 10 \mathrm{kV}$ power transformer from a distribution station, during a single phase short-circuit. For high voltage measurements, a power quality analyzer is used. At the same time a spectrum analyzer registered the associated electromagnetic compatibility issues.
\end{abstract}

The main conclusion that should be drawn is that conducted electromagnetic emissions represent phenomena that are compulsory accompanying short-circuits and they cannot be mitigated in their propagation path by the inductive filtering capacity of the subsequent power transformers solely, and they most often reach the low voltage equipment connected to the power system. Therefore, in order to achieve electromagnetic compatibility compliance, extra-filtering measures have to be taken.

\section{Key words}

electromagnetic compatibility, conducted emissions, power stations, earth fault, insertion loss, EMI filtering.

\section{Introduction}

Electromagnetic compatibility (EMC) is defined as the ability of an equipment or a system to satisfy three criteria:

- to cause no interference with other systems;

- to be not susceptible to emissions from other systems.

- to cause no interference with itself [1].
According to the International Electrotechnical Vocabulary Standard IEC/EN 60050-161, electromagnetic compatibility represents the ability of an equipment or system to function satisfactorily in its electromagnetic environment, without introducing intolerable electromagnetic disturbances to anything in that environment [2].

Terms as "satisfactory" and "intolerable" along with the syntagma of "electromagnetic environment", express a certain ambiguity, indicating the dependence on a given situation, but denoting on another hand a measure of the complexity of the domain to be covered. This complexity is rather difficult to be dispatched by a few limit values set by standards. Beyond the qualitative aspect of the definition, one must admit that the concept of electromagnetic compatibility, with all its facets, insinuates increasingly severe in the general concept of quality of life.

The lack of electromagnetic compatibility or a precarious solution of its issues might have several consequences, from annoyances determined by instance of a bad audio/video reception, to unforeseeable and unreliable operation of equipment in residential, tertiary or industrial environment and culminating with high risks upon the ecosystem and/or human life.

In most electromagnetic compatibility issues, one can notice an aggressor of the electromagnetic environment but in an equal measure a victim as well. The aggressor conducts or radiates a sufficient amount of electromagnetic energy, able to interfere with equipment representing a victim. On the other hand, the victim misoperates due to the interference with the aggressor or due to the electromagnetic fields present in its environment. 
According to [2] electromagnetic interference (EMI) represents the degradation of the performance of an equipment, transmission channel or system caused by an electromagnetic disturbance. Similarly, according to the same standard, electromagnetic disturbance represents any electromagnetic phenomenon which may degrade the performance of a device, equipment or system, or adversely affect living or inert matter. Note that the words "interference" and "disturbance" are often used indiscriminately.

Unfortunately, "victims" are not always completely innocents and that is why a poor electromagnetic compatibility design of equipment, can make them sensitive to the environmental electromagnetic fields and highly susceptible to electromagnetic noise, which represents time-varying electromagnetic phenomenon, apparently not conveying information and which may be superimposed on or combined with a wanted signal.

The specific regulations regarding electromagnetic compatibility, divide the electromagnetic interference mechanisms into two categories, namely conducted and radiated interference.

Conducted interference consists in the unwanted energy of the signals that leaves a certain product via its cables. For instance, the high frequency energy can couple to the supply source or to other adjacent products via the supply grid. High frequency signals can also escape via cables and radiate quite firmly. Conducted emissions are mainly controlled by filtering techniques and/or suitable grounding (earthing) practices.

Unfortunately, with respect to the radiated interference, in most of situations the literature doesn't differentiate between near and far field radiations, both categories being treated globally as radiated emissions. While the specialists in electromagnetism are familiar with this crucial distinction between the coupling in near field and propagation in far field, the vague terminology from the EMC regulations, tends to lead to confusions.

For the sake of simplicity, practical regulations consecrate the idea that radiated emissions are supposed predominant above the frequency of $30 \mathrm{MHz}$, while the conducted emissions are dominating the spectrum at frequencies lower than $30 \mathrm{MHz}$. It is obvious that nothing magical occurs at that border, but generally at frequencies beyond $30 \mathrm{MHz}$ the cables presents resonant frequencies, which may determine anomalies in conducted emissions measurements. At the same time, when frequency increases, cables become less efficient as a propagation medium, the dominating mode being the radiation from equipment or from cables.

Achieving compliance with electromagnetic compatibility means mainly to identify the sources of electromagnetic interference along with their coupling paths: galvanic (conductive) coupling, capacitive (electric) coupling, magnetic (inductive) coupling and electromagnetic radiation coupling. Only after that, the interference mitigation methods should be set. Depending on the source and on the coupling mechanism, the electromagnetic compatibility compliance may be obtained by filtering, by changing the geometry of the system or by shielding. On the other hand, regarding power quality issues, the compliance with the standards may be obtained using active, passive or hybrid filtering techniques.

Frequently, the concept of electric power quality and electromagnetic compatibility are confused. Over the course of time, an artificial dichotomy was created between the two concepts, even though the issues determined by the lack of power quality and of electromagnetic compatibility are fairly similar.

In literature one can find plenty and sometimes conflicting definitions of power quality, related more or less to the performance of equipment or to the possibility of measuring and quantifying the performance of the power system (see the IEEE Standards and the IEC and EN Standards). For instance the Council of European Energy Regulators-Working Group on Quality of Electricity Supply speaks about voltage quality and includes the following disturbances: "frequency, voltage magnitude and its variation, voltage dips, temporary and transient overvoltage, and harmonic distortion", without mentioning explicitly "current quality", which probably is implicitly considered where it affects the voltage quality [3]. The point of view here is again that current quality is only a concern only if it affects the voltage quality.

The difficulty of distinguishing between voltage and current disturbances is one of the reasons the term power quality is generally used. The term voltage quality is reserved for cases where only the voltage at a certain location is considered. The term current quality is generally used to describe the performance of power electronic converters connected to the power network [4]. In [5], the false dichotomy between power quality and electromagnetic compatibility is presented.

\section{Experimental results}

Power quality measurements were carried out in a $110 / 10$ $\mathrm{kV}$ power transformer and distribution station, which schematics diagram is presented in Fig. 1.

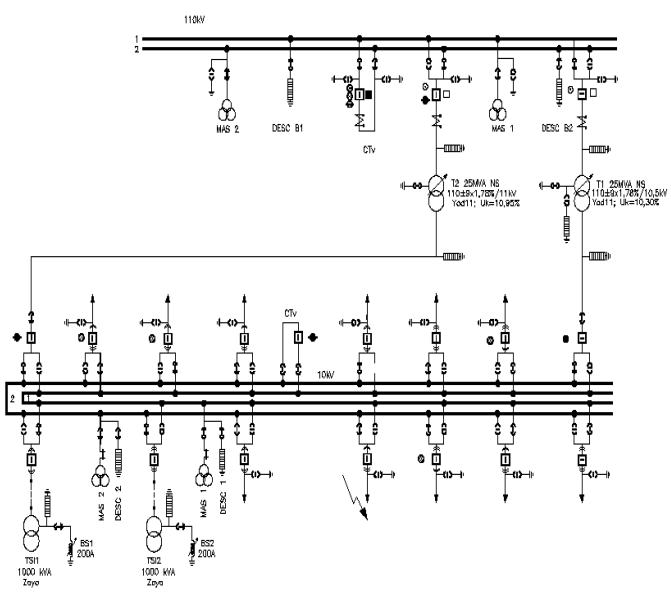

Fig. 3. Wiring diagram of the transformer station. 
In normal operation, the double bus arrangement power station works with the transformer $\mathrm{T} 1$ connected on the $110 \mathrm{kV}$ busbar no. 2 and generating power on the $10 \mathrm{kV}$ busbar no. 2. The second transformer T2 rests in standby. Both transformers have an apparent power of $25 \mathrm{MVA}$ at a voltage ratio of $1109 \times 1.78 \% / 10.5 \mathrm{kV}$. Two of the medium voltage outgoing feeders are connected to 1000 $\mathrm{kVA}$ transformers with the voltage ratio $10.5 / 0.4 \mathrm{~V}$, the others being distribution outgoing feeders. The other typical elements, such as circuit breakers, current and voltage transformers, disconnect and ground switches, surge arresters, etc., are also visible. The medium voltage cells are provided with overcurrent main and standby protection, performed with classic relay or digital relay. (see [6] for details).

In order to perform power quality measurements, typical power quality meters/analyzers and analyzers having the following functionalities are used:

- Data logging - capturing waveforms in real-time for later display;

- The ability to trigger on power-quality events such as sags, swells, or transients;

- Calculation of power-quality metrics such as total harmonic distortion in real-time;

- Spectrum analysis;

- Inputs for high-voltage probes and high-current probes.

In the experimental measurements, the Dranetz-BMI PowerXplorer PX5, a portable, hand-held, eight-channel power quality meter/monitor, which can survey, record and display data on four voltage channels and four current channels simultaneously, was used [7].

The PX5 firmware can monitor power quality phenomena for troubleshooting and/or compliance purposes. It can record inrush conditions, carry out long-term statistical studies to establish performance baselines, and perform field-based equipment testing and evaluation for commissioning and maintenance.

The firmware integrates an intuitive instrument setup procedure to ensure the capture of all relevant data for additional post process analysis, report writing, and data archiving using other compatible Dranetz-BMI software applications such as NodeLink and DranView. All PX5 functions are operable using a color LCD touch screen technology:

- Meter mode functions as a true rms voltmeter and a true rms clamp-on ammeter. Voltage and current measurements, along with other calculated and advanced power parameters, are displayed on the Meter mode screens in both textual and graphical format.

- Harmonics mode displays the amplitude and phase of each harmonic to the 63rd harmonic in both graphical and textual format.

- The phasor screen displays a graph that indicates phase relations between voltage and current based upon the angles at the fundamental frequency, as determined by Fourier analysis. Phasor diagram displays voltage and current phasors for all channels. Functioning as a phase angle meter, the unit can display system imbalance conditions and provides such information in textual form also. The phase angle display can also verify if monitoring connections have been made correctly.

- Flicker is a phenomenon due primarily to rapid small fluctuations of the voltage. Loads that exhibit continuous, rapid variations in the load current, particularly the reactive component, can cause voltage variations often referred to as flicker. Flicker is characterized by modulation at a frequency typically less than $25 \mathrm{~Hz}$. Modulating signal magnitudes as low as $0.5 \%$ of the fundamental for frequencies between $5-10 \mathrm{~Hz}$ can result in perceptible light flicker.

- An event occurs when a programmed threshold limit is crossed. An event consists of the pretrigger cycle(s), trigger cycle(s), and post-trigger cycle(s).

PX5 can monitor the following power configurations:

- $\quad$ Single Phase

- 3 Phase 2-Watt Meter Delta

- $\quad$ Split Phase

- Generic

- 3 Phase Delta

- $21 / 2$ Element without $V_{B}$

- 3 Phase Wye

- 2 1/2 Element without $\mathrm{V}_{\mathrm{C}}$

\section{A. Failure in a medium voltage power system}

The power quality analyzer was used to record the events for a single-phase short-circuit. Events are detailed in Figs. 2-4. Fig. 2 shows the waveforms at the moment that marks the start of the earth short-circuit in phase B. Similarly, Fig. 3 depicts the RMS values of the voltages in the three-phase distribution feeder.

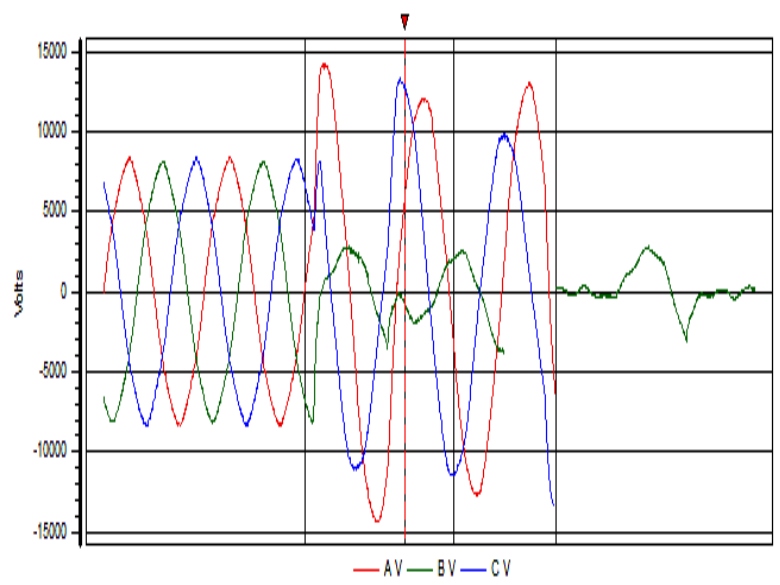

Fig. 2. Waveforms at the occurrence of the single fault (phase B).

The results depicted in Figs. 2-4, along with the values of the voltage presented in Table 1, permit to observe the evolution of the voltages in the very short period of time (approximately 40 seconds), until the fault in phase B was cleared by the protection system. Obviously, in cases like this, the protection system must trigger within a 
reasonable time interval in order to protect the conductors of thermal and dynamic stress.

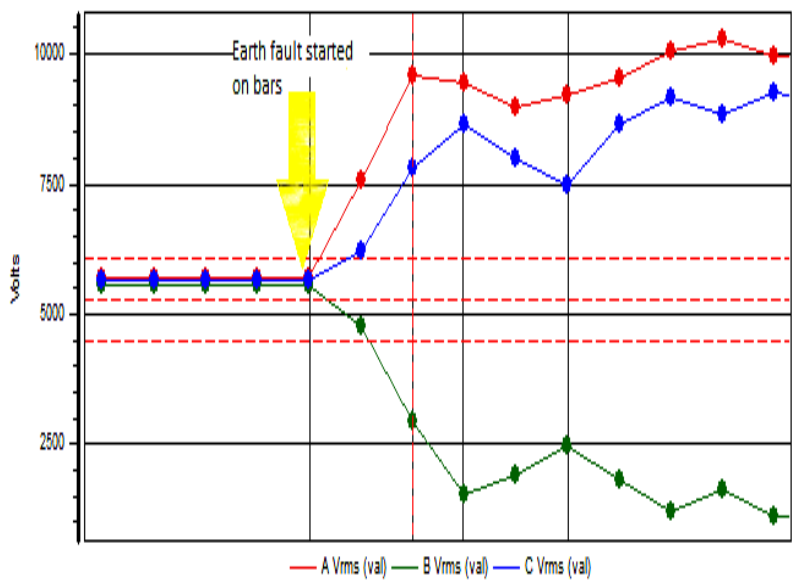

Fig. 3. RMS values at the occurrence of the single fault (phase B).

Table I. - RMS and peak values at the occurrence of the single phase fault.

\begin{tabular}{|l|l|c|c|}
\hline & \multicolumn{1}{|c|}{$\mathrm{A}$} & $\mathrm{B}$ & $\mathrm{C}$ \\
\hline $\mathrm{V}_{\text {rms }}$ & $9480 \mathrm{~V}$ & $1537 \mathrm{~V}$ & $8629 \mathrm{~V}$ \\
\hline $\mathrm{V}_{\text {peak }}$ & $14343 \mathrm{~V}$ & $3510 \mathrm{~V}$ & $13293 \mathrm{~V}$ \\
\hline
\end{tabular}
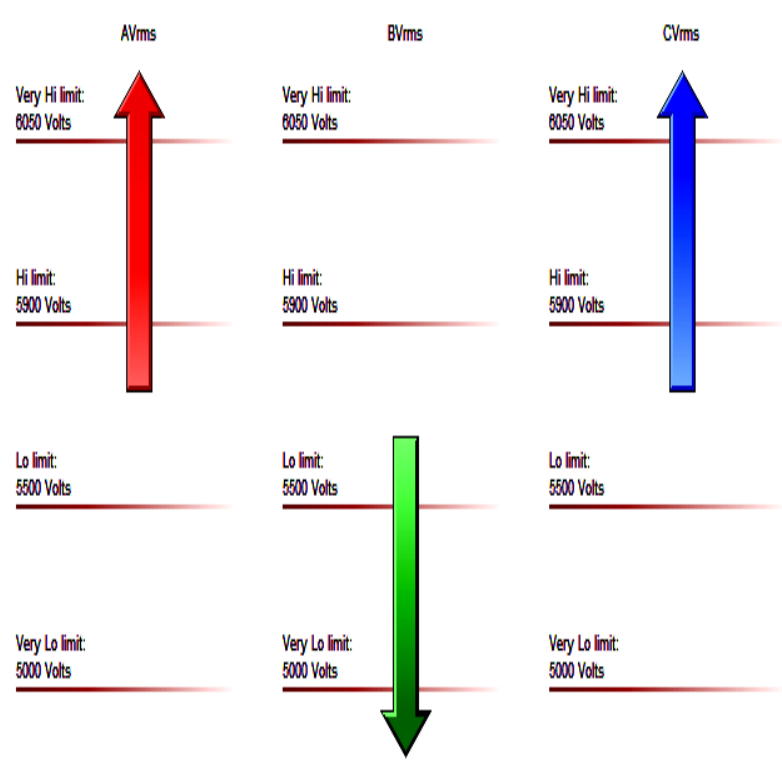

Lo limit:
5500 Volts

Very Lo imit:

5000 Volts

Fig. 4. Trend of voltages at the occurrence of the single fault (phase B).

\section{B. Conducted electromagnetic interference issues}

Conducted electromagnetic interference was analyzed in the adjacent "alleged healthy" distribution feeder before and during the occurrence of the single phase short circuit. Measurements were performed using a measurement setup made of a line impedance stabilization network (LISN) and a spectrum analyzer.

Basically, a LISN must present constant impedance to the product's power cord outlet over the frequency range of the conducted emission test. Inside the LISN the power lines are terminated with a well-defined impedance network, against each other and against ground, LISN being in principle a filter network.

The two major objectives of the LISN are: to present constant impedance $(50 \Omega)$ between the phase conductor and the safety wire and between the neutral conductor and the safety wire and to prevent external conducted noise on the power system net from contaminating the measurement. These two objectives are to be satisfied only over the frequency range of the conducted emission test $(100 \mathrm{kHz}-30 \mathrm{MHz})$. Another requirement for the LISN is to allow the $50 \mathrm{~Hz}(60 \mathrm{~Hz})$ power required for the proper product's operation.

Spectrum analyzers can be an alternative of EMC measuring receivers, widely used for "quick-look" testing and diagnosis [1]. They are basically radio receivers having a bandpass filter that is swept in time. Measurements were performed using a HM 6050-2 LISN and a HM 5014 spectrum analyzer.

In order to display the spectral amplitudes, detection of the input signal is needed. In this respect, three types of detectors of radiofrequency emissions can be used. Their features are depicted in the standard CISPR 16-1-1:2015 RLV [8].

a. Peak detector, which responds with an extremely short time constant for charging and a very long time constant for discharging. Its schematics, for a sine input signal is presented in Fig. 5.
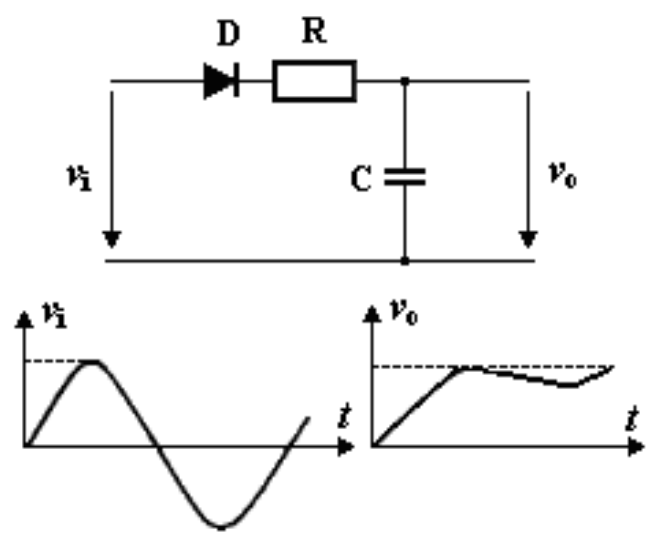

Fig. 5. Peak detector

b. Quasipeak detector, which has a short charging time constant which allows a fast charging, and a relatively long discharging time constant. The pulse repetition frequency (PRF) will have a significant influence on the QP-reading; the more pulses appearing within a certain time, the higher the QP-reading will be. Fig. 6 presents the simplified schematics and its associated waveforms.

c. Average detector, which indicates proportional to the pulse repetition frequency. This can be expressed as an increase in AV-reading by $20 \mathrm{~dB}$ if the PRF increases by factor 10 ( 1 decade). It also corresponds to an increase by $6 \mathrm{~dB}$ if the 
PRF is doubled (octave). The schematics and its associated waveforms are presented in Fig. 7.
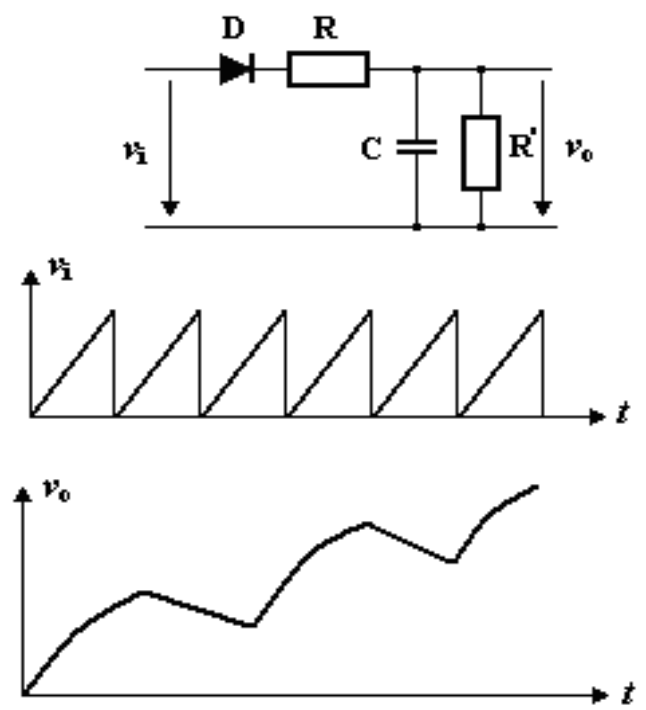

Fig. 6. Quasipeak detector

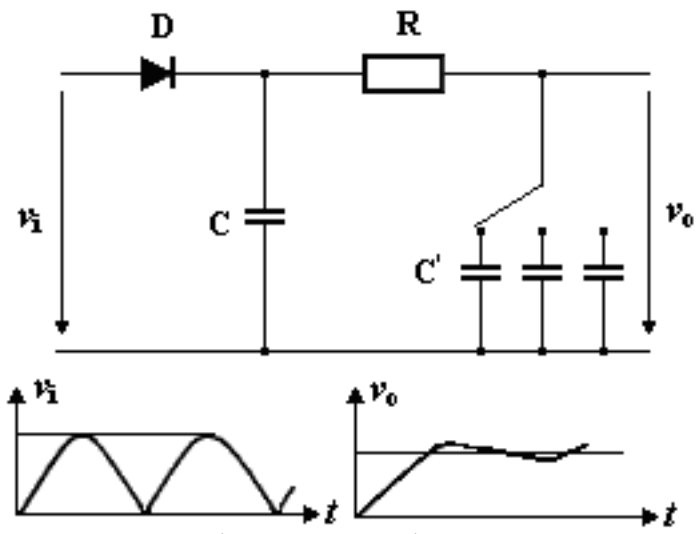

Fig. 7. Average detector

Finally, the RMS detector indicates proportional to the square root of the pulse repetition frequency. This corresponds to a RMS reading which increases by $10 \mathrm{~dB}$ when the PRF increases factor 10 (1 decade). It can also be expressed as an increase by $3 \mathrm{~dB}$ per octave (factor 2 ) of the PRF.

An alternative to the hardware detectors is a software computation of those parameters (for HM 5014 the firmware AS $100 \mathrm{E}$ is available).

The standard EN 55011:2016 set the limits of conducted emissions in terms of quasipeak (QP - red line) and average (AV - blue line) values as it is depicted in Fig. 8.

In normal operation of the power station, in the analyzed outgoing feeder the conducted emissions are inside the peak and average limits set by the standard EN 55011:2016 [9], with all the three significant values.

In Fig. 9 one can see that the root mean square (RMS), quasipeak (PQ) and average (AV) values are almost 20 $\mathrm{dB} \mu \mathrm{V}$ below the limits set by the standard. The RMS value is displayed in green, while the QP value is displayed in red and finally the AV value in blue.
At the moment of the earth fault in the adjacent distribution feeder, things are changing, and the quasipeak value overcomes the value set by the standard by almost $20 \mathrm{~dB} \mu \mathrm{V}$, as one can observe in Fig. 10.

The phenomenon is similar to the one presented in [5] and is due to magnetic coupling in near field, being prone to lead to damage or to a temporary misoperation of the equipment connected thereto.

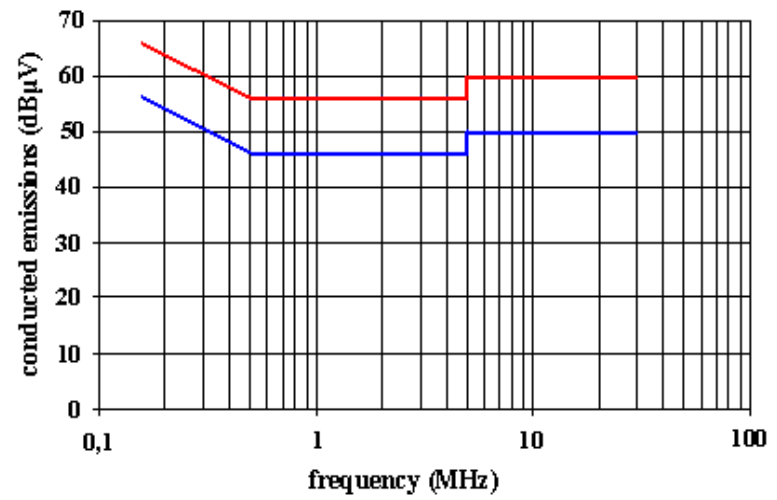

Fig. 8. Limit values of QP and AV values set by the standard EN 55011:2016

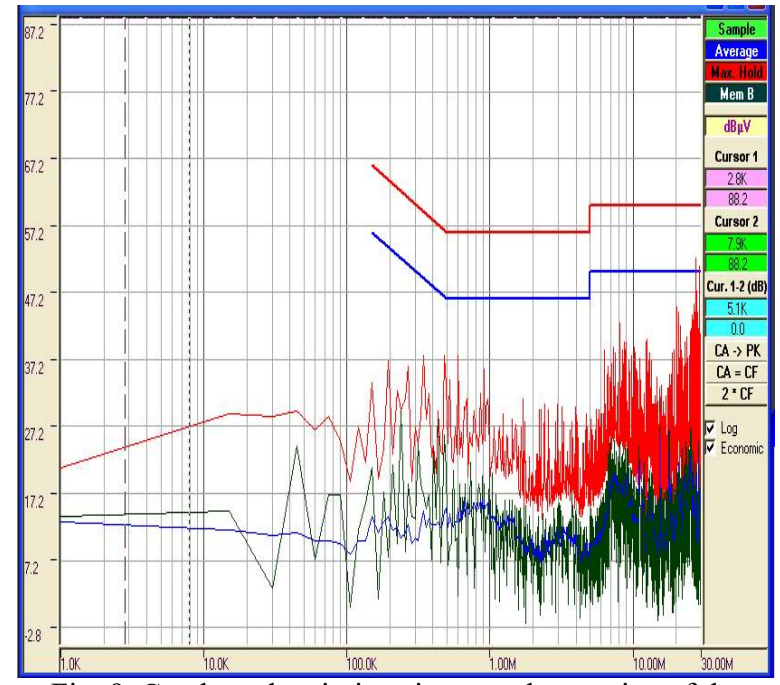

Fig. 9. Conducted emissions in normal operation of the power station

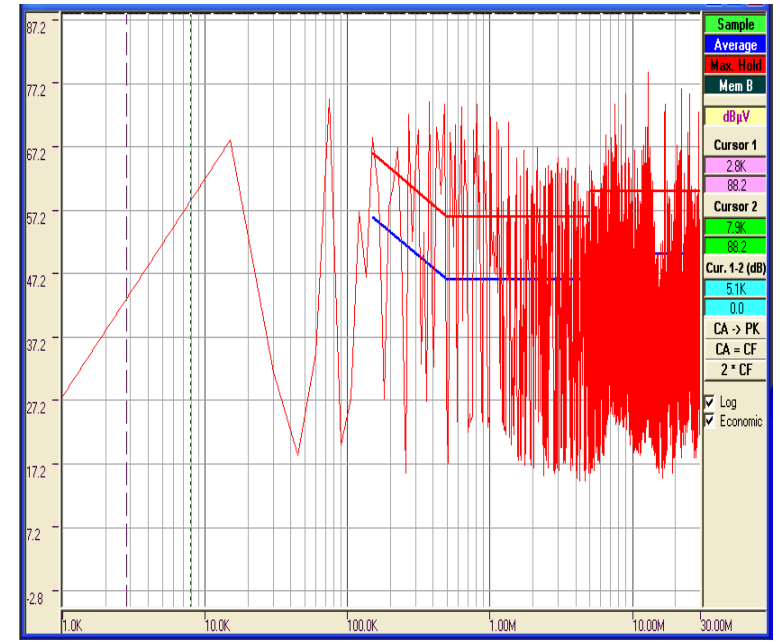

Fig. 10. Conducted emissions in the moment of the single phase short-circuit 
One could say that the case studied was a medium voltage one and, until reaching low voltage, the conducted emissions have to pass through at least one subsequent power transformer which acts like a low pass filter, which mitigates perturbations. It is true that the filtering ability of transformers for high frequency signals is quite well known due to the increasing inductive reactance ( $\left.X_{L}=2 \pi f \cdot L\right)$. However, in higher frequencies, the ferromagnetic material of the magnetic circuit of power transformers is no more effective.

In the analysis of higher frequency phenomena, one must rely on the complex permeability of magnetic materials: $\underline{\mu}=\mu^{\prime}-j \mu^{\prime \prime}$ which leads to the following equation for the insertion loss, which represents the main value characterizing EMI filtration $|\underline{Z}|=\sqrt{R^{2}+X^{2}}$. The components of the insertion loss are $R=2 \pi f \cdot L_{0} \cdot \mu^{\prime \prime}$ respectively $X=2 \pi f \cdot L_{0} \cdot \mu^{\prime}[10]$.

Figs. 11 - 12 display the two components of the insertion loss for the most common magnetic materials used in EMI mitigation, the resistive and the reactive parts. It can be clearly seen that the insertion loss of ferromagnetic materials becomes zero beyond $10 \mathrm{MHz}$. This limit is not sufficient to mitigate electromagnetic conducted emissions and in these cases extra-filtering measures have to be taken.

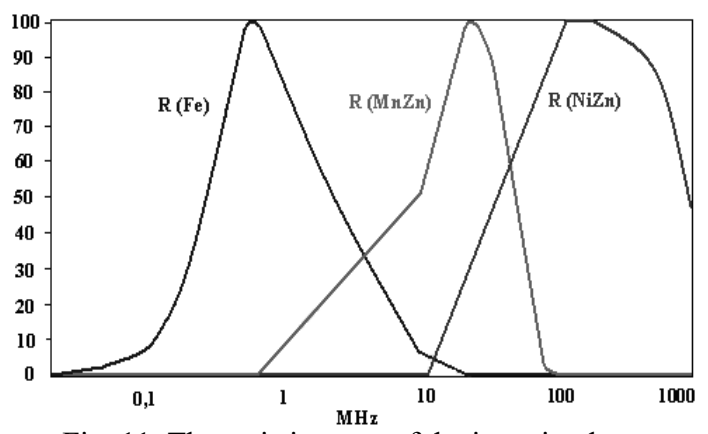

Fig. 11. The resistive part of the insertion loss

It is known that EMI mitigation is obtained with common mode chokes made of MnZn or NiZn ferrites, having an adequate magnetic permeability at higher frequencies [11].

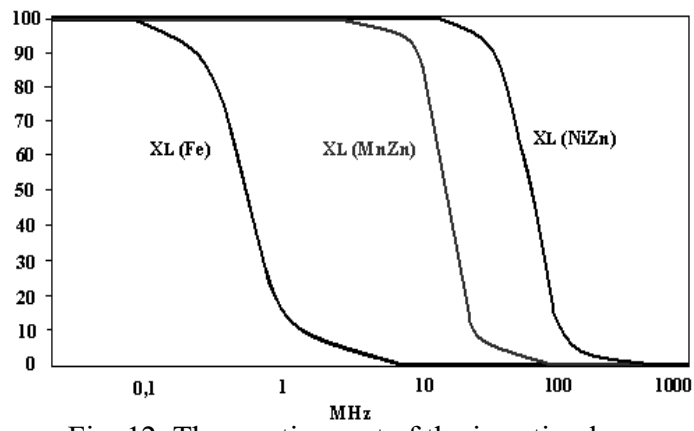

Fig. 12. The reactive part of the insertion loss

\section{Conclusions}

In conclusion, the short-circuit that occurred in the medium voltage outgoing feeder induced conducted emissions in the healthy adjacent one. These conducted electromagnetic emissions are prone to damage sensitive low voltage equipment connected thereto, or at least may lead to their temporary misoperation.

\section{References}

[1] Paul, C.R., Introduction to Electromagnetic Compatibility, Second Edition, John Wiley \& Sons, Inc., 2006, p.p. 1-3.

[2] *** IEC 60050-161:1990/AMD7:2017, International Electrotechnical Vocabulary - Part 161: Electromagnetic compatibility

[3] Council of European Energy Regulators-Working Group on Quality of Electricity Supply (2001), Quality of electricity supply: Initial benchmarking on actual levels, standards and regulatory strategies.

[4] Bollen, M. H. J., Yu-Hua Gu, I., Signal Processing Of Power Quality Disturbances, John Wiley \& Sons, Inc., 2006.

[5] Perez Donsion, M., Buzdugan, M.I., Power Quality, Cambridge Scholars Publishing, 2016, pp 2-18.

[6] Varodi, T. et al., Monitoring 110/10 kV Power Station -case study, Acta Electrotechnica, vol. 54, no. 5, pp. 522-525, Mediamira Science Publisher, Cluj-Napoca, 2013.

[7] *** Dranetz BMI, PowerXplorer PX5, User's Guide.

[8] *** CISPR 16-1-1:2015 RLV, Specification for radio disturbance and immunity measuring apparatus and methods Part 1-1: Radio disturbance and immunity measuring apparatus Measuring apparatus.

[9] ${ }^{* * *}$ EN 55011:2016 Industrial, scientific and medical equipment - Radio frequency disturbance characteristics; limits and methods of measurement.

[10] Gerfer, A.,Ralf, B., Zenker,H., Trilogy of Inductors, Swiridoff Verlag, 2002.

[11] Buzdugan, M.I., Bălan, H., Buzdugan, T.I., Some Procedures in Mitigating Conducted Electromagnetic Interference, ICREPQ'11, Las Palmas de Gran Canaria (Spain), April, 2011. 